\title{
Design and implementation of feeder automation
}

\author{
Liu Penghou \\ Qingdao University of Technology, 266300
}

\begin{abstract}
With the development of national economy, the demand for electricity from all walks of life has been gradually increased. People also put forward higher requests to the reliability of power supply. Feeder automation can quickly cut off the faulty line to ensure the safe and stable operation of the line. Under the sustainable development of modern society, people attach a great importance to all aspects of smart power grid construction, which is also significant for the smart grid distribution work. To ensure the reliable and high-quality power supply environment, the scientific and reasonable design and planning of power distribution automation system shall pay attention to arranging power distribution, and the appropriate feeder automation operation mode shall be selected. Only in this way can we ensure that the power outage is short once a fault happening in power grid system. At the same time, the line loss rate can be reduced, the efficiency of power supply can be raised, and issues in power equipment investment can be effectively treated. This paper mainly discusses the design and implementation of feeder automation, puts forward some practical application measures, and provides reference for the stable operation of feeder automation.
\end{abstract}

\section{INTRODUCTION}

The developing national economy has promoted the further development of power system modernization, and the continuous improvement and development of distribution network automation system have been the development trend of modern power enterprises. The monitoring management function equipped with the distribution network automation system of control backstage has an excellent integration and perfection. The feeder automation and the combination of intelligent terminal can effectively measure the operation results of distribution network automation system. The continuous development of power system makes the problems of distribution network become more and more serious, and the development of power demand and power facilities is not coordinated. For instance, we can easily find the problem in recovery, processing and load of distribution network fault, which cannot adapt to the needs of the development of modern society. Therefore, it is of great practical significance for the safe operation of feeder automation to discuss the design and implementation of feeder automation.

\section{PRINCIPLES OF DISTRIBUTION NETWORK AUTOMATION SYSTEM}

\subsection{Principles of fault diagnosis}

Whether there is any fault current passing through the switches on feeder line, or we can determine it based on the judgment of other fault signals. If the fault in the feeder line is single, the fault section is the power supply in the last switch side direction that flows from the fault current to the end, and the first switch section that did not flow any fault current is important.

\subsection{Failure estimation algorithm}

Distribution main station system constitutes its elements by using distribution network defined by the user, such as the relationship between power point, the subsection switch, feeder section, sectional switch and power point connection. Afterwards, matrix for describing the relation of distribution network topology structure shall be automatically generated. Then use various fault signals collected by SCADA system that flow through the segmentation switch to ensure the timely formation of the appropriate fault matrix. Results obtained by multiplying the two matrices shall be normalized, then the fault section shall be accurately located.

\subsection{Starting conditions of failure estimation algorithm}

Distribution main station system uses SCADA system to collect the state of the outlet switch of each power point and the total signals of the fault. As a result, if the power point is faulty and the tripping problem occurs, the fault location algorithm module can be started immediately. 


\subsection{Power restoration by distribution main station system}

When the distribution network system fails, the power switch will trip, which makes the non fault lines outage. However, in order to ensure the normal and stable operation of the circuit, and the improvement of the safety and efficiency of power supply, we need to resume power supply of non fault lines in time. Distribution main station system puts forward relevant ways to restore power, and restore power supply automatically and manually by combining specific problems with graphs.

\section{DESIGN OF FEEDING SYSTEM IN DISTRIBUTION NETWORK}

\subsection{Design scheme}

The design scheme should be based on the following aspects in designing feeding system in distribution network:

(1) Layers of master station that generally receives FTU terminal data, in which the integration work of SCADA system data and FTU terminal data are very critical. We can use the network hardware equipment to realize the integration of two kinds of data, but the software system shall be built based on Windows/UNIX hybrid platform, so as to carry out deep analysis of all kinds of information in the system, monitor system fault conditions in real time, and carry out effective coordination of all aspects of the work of the power. In realizing the system optimization, layers of master station should focus on improving the application functions and functional modules, storing the data in the relevant functional modules, and generating reports with relevant data by graphics and model based software, which shall make it possible for the user to access the information at any time.

(2) Slave station of distribution network automation. In designing slave station of distribution network automation, we should fully integrate the WEB server and the company MIS network so as to provide the corresponding WEB browsing service. Afterwards, we can use them in substation, the open and close operation, which can effectively control the WEB server in the information area, meanwhile collecting and filtering all kinds of information during this period. What's more, it will put forward the corresponding warning signal when the information being integrated, which shall provide CSOE, fault location and other functions for the operation of the distribution network, and achieve the purpose of fault isolation.

(3) Distribution terminal layer. In designing this layer, we should strictly integrate data information, and strictly screen information, then send the useful information. Traditional distribution network construction mode cannot meet the needs of modern social development, so we should build feeder automation system according to this, and create a good system to monitor the environment.

\subsection{Terminal design of fault detection}

We should focus on creating a good fault detection terminal environment when designing the feeder automation system. Sheathing materials should be PC/ABS alloy materials, which shall be put under the minus 60-120 degrees celsius, then electroplate it with TUNGKOUPC/ABC so as to improve fault detection. We should take single chip as the core in the terminal design of fault detection, whose operation speed is 5 million times per minute. Afterwards, carry on the sampling with 10 digits to judge the existence state of fault. We should use solar energy storage battery and supply power with lithium thionyl chloride battery to ensure the accuracy of the results, and power consumption shall not be more than $0.1 \mathrm{~mA}$ in dormancy. Use GSM network to collect and screen fault information, and effectively transfer it to supervision center to solve problems in the system. We should strictly analyze the data to determine whether the line is in the case of short circuit, or if the ground phase voltage drop is greater than $3 \mathrm{kV}$. Zero sequence current mutation is also greater than $10 \mathrm{~A}$, then some faults exist in the operation of the system. Determine whether the alarm signal needs to be issued according to the results, and if there is a power failure. If the voltage is 0 and the current is 0 , the power failure occurs in the monitoring points.

\subsection{Communication structure design}

GPRS has been widely used in major industries with its own economic advantages. When building the GPRS data communication mode, connect related terminal in communication structure, and pack the data into IP packets, then send GPRS to power system, send the data to SCADA. Finally, make reasonable decisions, and strictly implement terminal tasks after making a full analysis. Design of GPRS correspondence network should be connected with special-line and router, and transmission time delay should be strictly controlled within 1 second. Forward the corresponding data with the GPRS data center service system to form a perfect communication space.

\subsection{Automation system design}

We should focus on the following aspects in designing automation system:

(1) Regard GIS as master station layers of feeder automation, so as to ensure that it shall have telemetry, remote control, remote communication and other functions. What's more, regard intelligent permanent magnetic mechanism coincidence device as the basement so that when a fault occurs, GPS collects data about the voltage, current, power and composite power flow, at the same time, strictly implement the corresponding data instructions and carry out a comprehensive analysis of the differences between different states. As a result, make sure that the fault information in GIS can be timely feedback.

(2) Use SGSN to design mobile terminal, and use SGSN to send APN to HLR to query mobile terminal, then 
make a chart with the feedback data so that users can query related data on the network, at this point, we can form a one-to-many or many-to-many data delivery model.

(3) In designing automation system, we should combine GPRS with GGSN, and set APN point in GGSN, so as to achieve the purpose of passing private information data.

\section{IMPLEMENT OF FEEDER AUTOMATION}

\subsection{Fault in main loop}

If a fault occurs between systems, the outlet position of the substation will immediately trip, then re-close it. If the fault is transient, re-closing starts, while if the fault is a permanent fault, it will trip again, at the same time, it will be in a closed state. The information collected by the master station system is the basis of the network topology, by which we can quickly determine the fault section, and then send the remote command promptly, thus to obtain the purpose of opening and then closing up. We can achieve the purpose of separating and transferring power supply from 45 seconds to 60 seconds after determining the location of the fault. When a fault occurs in the relevant section of the main loop, the fault treatment process is similar to that of the above operation, thus it is OK to repeat the above operation.

\subsection{Fault in branch line}

If a fault occurs between systems, the circuit breaker in the front position of the outlet of the substation will take immediate actions. The substation outlet after tripping will produce returns, when the circuit breaker will overlap again. If the fault is transient, re-closing starts, while if the fault is a permanent fault, it will trip again, at the same time, it will be in a closed state. After the failure is cleared, it can run stably, thus there is no need to isolate and transfer the power supply.

Main loops of the distribution network transmission line of X city are equipped with load switches, and the branch circuit is equipped with the corresponding circuit breaker. The principle of dealing with the fault of the circuit structure is mainly that the branch line fault is eliminated by the tripping of branch line circuit breaker; while faults in main loop are controlled by centralized mode, so if there is a fault in the system, the substation will start tripping, thus to determine the location of the fault according to the information collected by the main station, and promptly issue a command to remove the fault as well as achieve the purpose of separation and the transferring of power supply. The design scheme is simple, and because master station involves little work, the overhead line fault handling can be fast and accurate. Table 1 refers to the project evaluation index of distribution feeder automation in power enterprises in $\mathrm{X}$ city.

Table 1 Project Evaluation Index of Distribution Feeder Automation in Power Enterprises in X City

\begin{tabular}{l|l}
\hline \multirow{5}{*}{ First-level indexes } & Secondary indexes \\
\cline { 2 - 2 } Secondary indexes & Output value per unit of electricity B11 \\
\cline { 2 - 2 } Technical efficiency index U2 & Power supply load net asset B12 \\
\cline { 2 - 2 } Social benefit index U3 & Cost of the unit substation capacity B13 \\
\cline { 2 - 2 } & Load ratio of main transformer B14 \\
\cline { 2 - 2 } & Average outage time of users B15 \\
\cline { 2 - 2 } & Voltage deviation B16 \\
\cline { 2 - 2 } & One family one standard rate B17 \\
\cline { 2 - 2 } & Power consumption rate B18 \\
\hline
\end{tabular}

This is the project evaluation index of distribution feeder automation in power enterprises in X city, from which we can find that when it comes to the popularization and application of feeder automation, people lay stress on the output value per unit of electricity, cost of the unit substation capacity, cost of unit capacity and load ratio of main transformer and regard them as important evaluation indexes, so as to create a stable supply space; while feeding system in distribution network operates, the corresponding fault state variables are all installed in the intelligent terminal unit. If the fault current exceeds the setting value, the state variables shall be represented as 1 , and if the fault current is less than the value of the whole set, the state variable shall be represented as 0 . What's more, the system protection end will give the variable feedback to the terminal timely where fault location is analyzed after changing the number of state parameters, and then immediately send trip command, after which protection system can be activated. When judging the state variables, we can transmit information through GPRS, calculate the related power and voltage value, and take corresponding protection measures. What's more, we should improve the operating efficiency of feeder automation to ensure stable operation of power system.

\section{CONCLUSIONS}

With the rapid development of modern society and economy, the level of information technology has also been promoted, and traditional feeder design of power enterprises cannot meet the needs of modern social development. Therefore, in order to create a good environment for of distribution network, we should pay much attention to the design of feeder automation, and we should focus on the strict analysis on automation system structure, system communication structure, fault detection terminal, etc., so as to ensure that the design is scientific and effective, scientifically control problems in the distribution, and ensure the safe and stable operation of distribution network. In addition, we should rapidly 
remove all the faults occurring in the operation of feeder system to improve operating efficiency.

\section{REFERENCES}

1. Pan Qing. Exploration on realization of automatic line fault isolation function of distribution network automation system[J]. Electrical Applications, 2012(8).

2. Fu Xichen. Analysis and Application of Distribution Network Automation in Isolating Distribution Network Fault[J].Technology Wind, 2015(24).

3. Zheng Liji. Discussion on Common Problems of Planning and Construction of Distribution Network Automation[J]. Straits Science, 011(11).

4. $\mathrm{Du}$ Haodong. Analysis and Research on Communication Technology of Distribution Network Automation[J]. China New Telecommunications, 2013(14).

5. Li Jiaxu, Gao Mingbo. Design and Implementation of Distribution Network Automation System[J]. Bengang Technology, 2015(02).

6. Chen Lei, Liu Wei. Design and Implementation of Distribution Network Automation System in Tiedong Substation[J]. Coal Mine Modernization, 2011(02).

7. Peng Hui, Ren Yuan, Song Xin, Chen Ning, Ge Yiyong. System Design of Distributed Integrated with County Technical Support Based on Dual Core Architecture[J]. Automation of Electric Power Systems, 2013(04). 